\title{
Consistent histories for tunneling molecules subject to collisional decoherence
}

\author{
Patrick J. Coles, ${ }^{1,2}$ Vlad Gheorghiu, ${ }^{1,3}$ and Robert B. Griffiths ${ }^{1}$ \\ ${ }^{1}$ Department of Physics, Carnegie Mellon University, Pittsburgh, Pennsylvania 15213, USA \\ ${ }^{2}$ Centre for Quantum Technologies, National University of Singapore, Singapore \\ ${ }^{3}$ Institute for Quantum Information Science and Department of Mathematics and Statistics, \\ University of Calgary, Calgary, Alberta T2N 1N4, Canada
}

\begin{abstract}
The decoherence of a two-state tunneling molecule, such as a chiral molecule or ammonia, due to collisions with a buffer gas is analyzed in terms of a succession of quantum states of the molecule satisfying the conditions for a consistent family of histories. With $\hbar \omega$ the separation in energy of the levels in the isolated molecule and $\gamma$ a decoherence rate proportional to the rate of collisions, we find for $\gamma \gg \omega$ (strong decoherence) a consistent family in which the molecule flips randomly back and forth between the left- and right-handed chiral states in a stationary Markov process. For $\gamma<\omega$ there is a family in which the molecule oscillates continuously between the different chiral states, but with occasional random changes of phase, at a frequency that goes to zero at a phase transition $\gamma=\omega$. This transition is similar to the behavior of the inversion frequency of ammonia with increasing pressure, but will be difficult to observe in chiral molecules such as $\mathrm{D}_{2} \mathrm{~S}_{2}$. There are additional consistent families both for $\gamma>\omega$ and for $\gamma<\omega$. In addition we relate the speed with which chiral information is transferred to the environment to the rate of decrease of complementary types of information (e.g., parity information) remaining in the molecule itself.
\end{abstract}

\section{INTRODUCTION}

The decoherence produced by the interaction of a quantum system with its environment is ubiquitous in nature and plays an important role in current quantum theory in at least two ways. First, it is widely believed that decoherence helps understand how the classical physics of macroscopic objects emerges as an approximation to underlying quantum mechanical laws. Second, decoherence is the great enemy of quantum computation, quantum cryptography, and other schemes seeking to utilize specifically quantum effects for particular processes. For both reasons it is important to study specific microscopic models from which one can hope to obtain general principles for decoherence. The present paper is the study of a simple two-level system which can be thought of as a crude microscopic model of chiral molecules or ammonia in which the lowest quantum energy levels correspond to the nearly degenerate eigenstates of a double-well potential, with decoherence occuring through collisions with particles in the environment.

Microscopic studies of decoherence are often framed in terms of a master equation for the density operator of the decohering system. Such descriptions are perfectly valid, but because they represent the average of a large ensemble of nominally identical systems, each with a different specific time development, they provide less information and less physical insight than the actual history of a single system. For example, in the phenomenon of intermittent fluorescence a single ion in a trap shows intermittent light and dark periods when it does or does not scatter resonance radiation [1]. This behavior is not directly reflected in the density oper- ator, even though from the latter one can deduce parameters which govern the statistical behavior of the individual ion.

Another way to understand the limitations of the density operator description is to consider its classical analog for a Brownian particle confined to a small but macroscopic volume of a fluid by rigid walls. The probability distribution $\rho(\boldsymbol{r}, t)$ of the particle position $\boldsymbol{r}$ will eventually tend to a constant over the region accessible to the particle, whereas the particle itself will continue to exhibit a sort of random walk. More details of what is going on in this steady-state situation is provided by the joint probability distribution of the sequence of successive positions $\boldsymbol{r}_{1}, \boldsymbol{r}_{2}, \ldots$ of the particle at a sequence of times $t_{1}, t_{2}, \ldots$, that is, its history. Averaging over a large number of histories will yield $\rho(\boldsymbol{r}, t)$, but in the process the information needed for a more detailed temporal description of the particle is lost. In the quantum case unravelings of the master equation provide a more detailed description of the microscopic time development, but these are often viewed as mathematical artifacts having no necessary connection with what is really going on in the quantum system. There are many possible unravelings; which, if any are correct? Standing in the way of answering this question is the infamous measurement problem of quantum foundations: textbook quantum mechanics introduces probabilities by means of measurements, but cannot say what it actually is that is being measured.

However, the consistent histories or decoherent histories - hereafter simply referred to as histories formulation of quantum mechanics, has no measurement problem, and provides the tools needed to identify trajectories or sequences of events that actually correspond to physical processes. Or, putting it 
another way, it allows one to identify certain classes of microscopic stochastic processes which can be consistently described in a fully quantum mechanical terms. The histories approach has previously been applied to quantum optical systems by Brun [2, 3], though we believe the material presented here is the first application to the case of tunneling molecules, including chiral molecules.

Early in the development of quantum mechanics the question arose as to why chiral molecules are observed in left- and right-handed versions even though the quantum ground state should be a symmetrical combination of the two forms. Hund [4] provided the first step in addressing this paradox when he pointed out that the two enantiomers correspond to the two wells of a symmetrical potential with two minima, and that the time required to tunnel from one well to another for a typical chiral molecule is extremely long. A second step was provided by Simonius [ $\underline{5}$ ] who observed that interaction with the environment of a suitable sort (i.e., decoherence, though when he wrote that term was not yet current) can stabilize the chiral states for periods substantially longer than the tunneling time. At present it seems widely accepted that such decoherence is an important aspect of the stability of chiral molecules, though there have been dissenting voices, e.g. [6].

The time dependence of the two-state model introduced in Sec. III, when analyzed in terms of consistent histories using the principles discussed in Sec. III] and applied to specific consistent families in Sec. IV yields some insight into this stability problem. In particular, we find that if the rate of decoherence due to collisions $\gamma$ (a parameter in our model) is much larger than the tunneling rate $\omega$ in an isolated molecule, there is a consistent family in which the molecule spends a long but random period of time in each of the chiral states before flipping to the one of opposite chirality, in a two-state Markov process. As $\gamma$ decreases the flips become more rapid and the "dressed" quantum states between which the flips occur become less and less chiral, with this type of family finally disappearing at a phase transition $\gamma=\omega$. For $\gamma<\omega$ there is a different consistent family with a rapid but continuous oscillation of the molecule back and forth between its chiral states, interrupted at random times by a change in phase. There are a variety of other consistent families, and these are discussed, along with their physical interpretation, in Sec. IV] Most chiral molecules in most circumstances will be in the strong decoherence regime. We give some approximate numerical values in Sec. VIA for $\mathrm{D}_{2} \mathrm{~S}_{2}$ in a buffer gas of helium, as it has been the subject of some careful calculations in [7]. On the other hand the ammonia molecule, which though not itself a chiral molecule can behave like one in certain rotational states, has an inversion (tunneling) transition with a frequency that goes to zero with increasing pressure. This is probably an example of, or at least very similar to, the $\gamma=\omega$ phase transition, for reasons discussed in Sec. VIB.

An intuitive way of characterizing the stochastic time development in the histories formalism is to quantify the information dynamics, e.g., the loss over time of information about the system's initial state due to random hopping. Such information loss is ultimately connected to decoherence [8], which can alternatively be viewed as flow of information about the system to the environment [9], and in Sec. $[\mathrm{V}$ we illustrate the quantitative connection between these two views of decoherence for our model. In our model decoherence corresponds to a flow of chiral information-i.e., is the molecule left or right handed? - to the environment. In Sec. $\nabla$ we analyze this using quantitative measures defined in Sec. IIIC and compare the flow of chiral information to the environment with the decrease of complementary types of information (e.g., parity information) about the earlier state of the molecule that remain in the molecule itself at later times.

Our conclusions are summarized in Sec. VII which also indicates some ways in which the results reported here could be usefully extended. A few mathematical derivations and details are placed in appendices.

\section{MICROSCOPIC MODEL AND MASTER EQUATION}

\section{A. Double-well potential and collisions}

We consider a quantum system, the molecule, with a double-well potential in which the two lowest energy eigenstates, $|0\rangle$ (even parity) and $|1\rangle$ (odd parity), are sufficiently well separated in energy from all the higher levels that the latter can be ignored. The Hamiltonian is of the form

$$
H=(1 / 2) \hbar \omega Z,
$$

where $Z=|0\rangle\langle 0|-| 1\rangle\langle 1|$ is the Pauli operator $\sigma_{3}=$ $\sigma_{z}$, so the energy splitting between the levels is $\hbar \omega$. The linear combinations

$$
|R\rangle=(|0\rangle+|1\rangle) / \sqrt{2}, \quad|L\rangle=(|0\rangle-|1\rangle) / \sqrt{2},
$$

represent the right- and left-handed chiral forms of the molecule, or in ammonia the nitrogen on one or the other side of the plane formed by the hydrogens. In real molecules there are, of course, additional degrees of freedom-rotations, vibrations, etc. We are assuming that for our purposes these can be ignored, i.e., the Hilbert space can be approximated as a tensor product of these other degrees of freedom with 
the two levels representing the tunneling, with negligible coupling between them. Hence the isolated molecule can be thought of as oscillating or tunneling between the $|R\rangle$ and $|L\rangle$ states at an angular frequency $\omega$. In the Bloch sphere picture the kets $|R\rangle$ and $|L\rangle$ correspond to the points on the positive and negative $x$ axis, and the sphere rotates about the $z$ axis as time increases.

Next we assume the molecule collides randomly with other particles (atoms or molecules), and the duration of each collision is short compared to the times we are interested in. Successive collisions need not be independent of each other, but we assume that correlations die away rapidly after some correlation time $\tau_{c}$, which could be shorter than the average time between collisions in a dilute gas, but might be significantly longer in a dense gas or liquid. We will consider properties of the molecule at a succession of times $t_{0}, t_{1}, t_{2} \ldots$, where the $m$ 'th time interval, $\Delta t_{m}=t_{m+1}-t_{m}$ is always greater than $\tau_{c}$, and ideally should be significantly greater than $\tau_{c}$. That is, we are using a description which is coarsegrained in time; the importance of this will appear later. During the $m$ 'th time interval there may be zero or one or more collisions of other particles with the molecule, and different collisions can have different effects. We shall assume that the probability distribution for these collisions in a particular interval, both for the times at which they occur and the effects which they have on the molecule, are statistically independent of what happens in other intervals. Obviously this cannot be exactly correct, but on physical grounds it seems reasonable provided $\Delta t_{m}$ is not too short, which is why we assume that it is larger than $\tau_{c}$. In addition we assume, as is appropriate for a steady state situation, that this probability distribution depends only on the length $\Delta t_{m}$ of the interval, and not otherwise on $t_{m}$.

The next assumption is that at the beginning of a time interval of length $\Delta t$ the molecule and the environment can be adequately described, for the purposes of what happens next, as a tensor product of a molecule state and some density operator for the environment. ${ }^{1}$ The latter is the quantum analog of a probability distribution for incoming particles which might collide with the molecule during this time interval. This density operator can be "puri-

\footnotetext{
${ }^{1}$ Working out the general connection of decoherence with thermodynamic irreversibility and the properties of steady state is, at a fundamental level, an unsolved problem. Our hope is that its eventual resolution will justify present practice by the experts in both the quantum information and decoherence communities, whose example we are following here. We note that some detailed quantum mechanical treatments of decoherence for situations similar to ours can be found in, e.g., [10, 11] and references therein.
}

fied" by regarding it as arising from an entangled pure state between the environment and an auxiliary reference system, which we also take to be part of the environment. The overall time development of the molecule and the environment during the interval $\Delta t$ is then given by a unitary time development operator, corresponding to an appropriate Hamiltonian, acting on the system and environment, resulting in an isometry mapping the Hilbert space $\mathcal{H}_{M}$ of the molecule onto $\mathcal{H}_{M} \otimes \mathcal{H}_{E}$, where $\mathcal{H}_{E}$ is the Hilbert space of the environment. If one traces out the environment the result is a quantum operation or channel from $\mathcal{H}_{M}$ to itself: the channel input is the molecule at the beginning of the time interval $\Delta t$ and its output is the molecule at the end of this interval. It is represented by a completely-positive trace-preserving (CPTP) superoperator $\mathcal{T}(\Delta t)$ from the space $\hat{\mathcal{H}}_{M}$ of linear operators on $\mathcal{H}_{M}$ to itself. Tracing out the molecule instead of environment at the end of the time interval results in a corresponding CPTP map $\mathcal{T}^{c}(\Delta t)$ from $\hat{\mathcal{H}}_{M}$ to $\hat{\mathcal{H}}_{E}$, representing the complementary channel. See, for example, [12] for further details on how the direct and complementary channel are related to the isometry.

The assumption of statistical independence of successive time intervals, and that the environment is in a steady state, allows us to treat the interval from $t_{1}$ to $t_{2}$ in the same way as the interval from $t_{0}$ to $t_{1}$. Thus a succession of time intervals can be thought of, so far as the molecule is concerned, as a set of channels in series, with dynamics corresponding to an appropriate composition of the superoperators $\mathcal{T}\left(\Delta t_{m}\right)$ for the corresponding intervals.

The use of a superoperator $\mathcal{T}(\Delta t)$ that depends only on the length $\Delta t$ of the time interval may give rise to the misleading impression that we are assuming exactly the same number and type of collisions for any interval of length $\Delta t$. But this is not so. To understand why, consider a classical stochastic process for which the independence of successive time intervals justifies using a Markov model, and for simplicity assume that all the time intervals are of equal length. Let $j$ be a discrete index labeling molecule states at a single time, $j_{m}$ its value at the beginning of the $m$ 'th time interval, and $M^{(n)}\left(j^{\prime}, j\right)$ the Markov matrix for a transition $j \rightarrow j^{\prime}$ if precisely $n$ collisions occur in one time interval. The probability distribution for a collection of histories that all begin in the state $j_{1}$ conditioned on a specified set $n_{1}, n_{2}, \ldots n_{f}$ of numbers of collisions in the different intervals is

$$
\begin{aligned}
& \operatorname{Pr}\left(j_{1}, j_{2}, \ldots j_{f+1} \mid n_{1}, n_{2}, \ldots n_{f}\right)= \\
& M^{\left(n_{f}\right)}\left(j_{f+1}, j_{f}\right) \cdots M^{\left(n_{2}\right)}\left(j_{3}, j_{2}\right) M^{\left(n_{1}\right)}\left(j_{2}, j_{1}\right)
\end{aligned}
$$

On the other hand, if the sequence of collision numbers is not known, the probability not conditioned 
on this information is given by

$$
\begin{aligned}
& \operatorname{Pr}\left(j_{1}, j_{2}, \ldots j_{f+1}\right)= \\
& M^{(a v)}\left(j_{f+1}, j_{f}\right) \cdots M^{(a v)}\left(j_{3}, j_{2}\right) M^{(a v)}\left(j_{2}, j_{1}\right)
\end{aligned}
$$

where $M_{j^{\prime} j}^{(a v)}$ is the averaged Markov matrix,

$$
M^{(a v)}\left(j^{\prime}, j\right)=\sum_{n} \operatorname{Pr}(n) M^{(n)}\left(j^{\prime}, j\right),
$$

and $\operatorname{Pr}(n)$ the probability of $n$ collisions during a single time interval. In the quantum case the single superoperator $\mathcal{T}(\Delta t)$ is the analog of an averaged Markov matrix, and it will allow us to correctly compute the probability of a sequence of histories as long as we do not try and condition it on more detailed information about the initial state of the environment at the beginning of the time interval.

\section{B. Explicit form for the superoperator $\mathcal{T}$}

As noted above, $\mathcal{T}(\Delta t)$ only makes physical sense for $\Delta t$ greater than some correlation time $\tau_{c}$. Keeping this in mind, it is nonetheless very convenient to think of the argument of $\mathcal{T}(\Delta t)$ as a continuous variable, which we shall hereafter denote by $t$, thus $\mathcal{T}(t)$. This superoperator can be written in various ways, e.g., using Kraus operators or as a matrix using some basis of the operator space of a qubit. A convenient basis is provided by the Pauli operators: $\sigma_{0}=I, \sigma_{1}=X, \sigma_{2}=Y, \sigma_{3}=Z$, in terms of which we write

$$
\mathcal{T}(t) \sigma_{j}=\sum_{k} \mathbf{T}_{k j}(t) \sigma_{k},
$$

using a matrix $\mathbf{T}$ of real coefficients whose first row (because $\mathcal{T}$ is trace preserving) is $(1,0,0,0)$. The remaining rows constitute a collection of 12 (real) parameters which are only constrained by inequalities that ensure that $\mathcal{T}$ is completely positive. Applying $\mathcal{T}(t)$ to a density operator $\rho=\sum_{j} \boldsymbol{\rho}_{j} \sigma_{j}$ at $t=0$, with the coefficients $\left\{\boldsymbol{\rho}_{j}\right\}$ regarded as a column vector $\boldsymbol{\rho}$, results in a density operator $\bar{\rho}=\sum_{j} \overline{\boldsymbol{\rho}}_{j} \sigma_{j}$ at time $t$, where $\overline{\boldsymbol{\rho}}=\mathbf{T} \cdot \boldsymbol{\rho}$.

Rather than explore the entire parameter space, we have assumed that $\mathbf{T}$ has the particularly simple form

$$
\mathbf{T}(t)=e^{t \mathbf{S}}, \quad \mathbf{S}=\left(\begin{array}{cccc}
0 & 0 & 0 & 0 \\
0 & 0 & -\omega & 0 \\
0 & \omega & -2 \gamma & 0 \\
0 & 0 & 0 & -2 \gamma
\end{array}\right)
$$

where $\omega$ is the precession frequency for $|R\rangle$ to $|L\rangle$ and back again for the isolated molecule - the energy difference between $|0\rangle$ and $|1\rangle$ is $\hbar \omega$ - and $\gamma \geq 0$ is the rate of decoherence. Justification based on scattering theory for this form of $\mathbf{T}$ has been discussed in 7 . 10.

To see the motivation behind (7), first consider the case of the isolated molecule with no decoherence, $\gamma=0$. Then

$$
\mathbf{T}(t)=\mathbf{R}(t)=\left(\begin{array}{cccc}
1 & 0 & 0 & 0 \\
0 & \cos \omega t & -\sin \omega t & 0 \\
0 & \sin \omega t & \cos \omega t & 0 \\
0 & 0 & 0 & 1
\end{array}\right)
$$

corresponds to precession about the $z$ axis in a Bloch sphere picture. Next, suppose that $\omega=0$, so that only decoherence is present. Then

$$
\mathbf{T}(t) \approx \mathbf{D}(t)=\left(\begin{array}{cccc}
1 & 0 & 0 & 0 \\
0 & 1 & 0 & 0 \\
0 & 0 & 1-2 \gamma t & 0 \\
0 & 0 & 0 & 1-2 \gamma t
\end{array}\right)
$$

when $t$ is small, and

$$
\mathbf{T}(t)=\mathbf{D}(t) \cdot \mathbf{R}(t)+\mathbf{O}\left(t^{2}\right)=\mathbf{R}(t) \cdot \mathbf{D}(t)+\mathbf{O}\left(t^{2}\right) .
$$

with $\mathbf{O}\left(t^{2}\right)$ a second order correction. Thus (7) combines the competing effects of decoherence and the molecule's internal dynamics.

The motivation behind (9) is a simple physical picture in which if the environment is initially in the state $|E\rangle$ its interaction with the molecule during a collision corresponds to the unitary transformation

$$
\begin{aligned}
& |L\rangle \otimes|E\rangle \rightarrow|L\rangle \otimes\left(\sqrt{1-2 p}|E\rangle+\sqrt{2 p}\left|E^{\prime}\right\rangle\right), \\
& |R\rangle \otimes|E\rangle \rightarrow|R\rangle \otimes\left(\sqrt{1-2 p}|E\rangle+\sqrt{2 p}\left|E^{\prime \prime}\right\rangle\right),
\end{aligned}
$$

where the environment states $|E\rangle,\left|E^{\prime}\right\rangle$ and $\left|E^{\prime \prime}\right\rangle$ are orthonormal. The intuitive idea is that the distinction between $|L\rangle$ and $|R\rangle$ is carried off to the distinct environmental states $\left|E^{\prime}\right\rangle$ and $\left|E^{\prime \prime}\right\rangle$ with an amplitude that increases with $p$, a quantity lying between 0 and $1 / 2$ which is a measure of the effectiveness of the decoherence. The decohering effect is unchanged if $|E\rangle$ on the right side of (11) is replaced with any other state $|\bar{E}\rangle$ as long as it is orthogonal to $\left|E^{\prime}\right\rangle$ and $\left|E^{\prime \prime}\right\rangle$, i.e., if the alteration does not depend upon the difference between $|L\rangle$ and $|R\rangle$. One can represent the channel corresponding to (11) by three Kraus operators associated with $|E\rangle,\left|E^{\prime}\right\rangle$ and $\left|E^{\prime \prime}\right\rangle$, but an equally good form uses just two Kraus operators $\sqrt{1-p} I$ and $\sqrt{p} X$ corresponding to a "bit flip" channel in [13] p. 376. When $p=0$ there is no decoherence (a perfect channel) whereas for $p=1 / 2$ the collision "collapses" the molecule into either $|R\rangle$ or $|L\rangle$. If one sets $p=\gamma t$ the superoperator corresponding to the process (11) is given by (9), and this makes sense for $t$ of the order of the time between collisions. However, as noted above in Sec. II A the superoperator $\mathcal{T}(t)$ can appropriately represent a situation 
in which the number of collisions in the interval $t$ is a random quantity.

The matrix $\mathbf{T}(t)$ and the density operator $\boldsymbol{\rho}(t)$ thought of as a column vector satisfy the simple linear differential equations:

$$
\frac{d \mathbf{T}}{d t}=\mathbf{S} \cdot \mathbf{T}, \quad \frac{d \boldsymbol{\rho}}{d t}=\mathbf{S} \cdot \boldsymbol{\rho} .
$$

The second is equivalent to a master equation in Lindblad form

$$
\frac{d \rho}{d t}=-\mathrm{i}[H, \rho] / \hbar+\gamma(X \rho X-\rho),
$$

with $H / \hbar=\omega Z / 2$ as in (1).

Solutions to (12) and (13) can of course be expressed as linear combinations of exponentials of the form $e^{\lambda_{j} t}$, where

$$
\begin{gathered}
\lambda_{1}=0, \lambda_{2}=-\gamma+\xi, \lambda_{3}=-\gamma-\xi, \lambda_{4}=-2 \gamma \\
\xi=\sqrt{\gamma^{2}-\omega^{2}}
\end{gathered}
$$

are the eigenvalues of the matrix $\mathbf{S}$. Note that $\lambda_{2}$ and $\lambda_{3}$ occur in solutions of the form $e^{\lambda t}$ to the damped oscillator equation $d^{2} x / d t^{2}+2 \gamma d x / d t+\omega^{2} x=0$. Thus for $\gamma<\omega$ they are complex conjugates of each other lying on a circle of radius $\omega$ in the complex plane, corresponding to oscillatory solutions, while for $\gamma>\omega$ both are real and negative, corresponding to damped motion without oscillation. Critical damping $\gamma=\omega$ corresponds to a phase transition in the sense of a changeover between two qualitatively different types of behavior. The explicit form of $\mathbf{T}(t)$ is given in Appendix A.

\section{GENERAL ASPECTS OF CONSISTENT HISTORIES AND INFORMATION}

\section{A. Introduction to histories}

In the (consistent or decoherent) histories formalism a history is a sequence of quantum properties, identified by projectors onto appropriate subspaces of the quantum Hilbert space, at a succession of times $t_{1}<t_{2}<\cdots<t_{f}$; see Ch. 8 of [14]. In the situation at hand we use a sample space of mutually exclusive histories formed by assuming that at time $t_{m}$ the properties of interest to us correspond to a collection $\left\{P_{m}^{\alpha_{m}}\right\}$ of projectors which form a decomposition of the identity:

$$
\sum_{\alpha_{m}} P_{m}^{\alpha_{m}}=I, \quad\left(P_{m}^{\alpha_{m}}\right)^{\dagger}=P_{m}^{\alpha_{m}}=\left(P_{m}^{\alpha_{m}}\right)^{2} .
$$

Here the subscript $m$ labels the time, while the superscript $\alpha_{m}$ is not an exponent but instead a label to differentiate the projectors at this time. Choosing at each time a property from the corresponding decomposition of the identity yields a history represented by a projector

$$
Y^{\boldsymbol{\alpha}}=P_{1}^{\alpha_{1}} \odot P_{2}^{\alpha_{2}} \odot \cdots P_{f}^{\alpha_{f}}, \quad \boldsymbol{\alpha}=\left(\alpha_{1}, \alpha_{2}, \ldots \alpha_{f}\right)
$$

on the history Hilbert space $\breve{\mathcal{H}}=\mathcal{H}^{\odot f}=\mathcal{H} \odot \mathcal{H} \odot$ ‥ $\mathcal{H}$ formed by the tensor product of the Hilbert space with itself $f$ times. Here $\odot$ is a tensor product symbol with the same significance as $\otimes$, but employed to distinguish different times. The physical significance of $Y^{\alpha}$ can be seen by reading (17) as "property $P_{1}^{\alpha_{1}}$ at time $t_{1}$ followed by property $P_{2}^{\alpha_{2}}$ at time $t_{2}$ followed by...."

For a closed system in which the unitary (Schrödinger) time development from $t_{m}$ to $t_{m+1}$ is described by the operator $U_{m+1, m}$, probabilities (probabilistic weights) can be assigned using the $d e-$ coherence functional 14]

$$
\begin{array}{r}
\mathbb{D}\left(Y^{\boldsymbol{\alpha}}, Y^{\boldsymbol{\beta}}\right)=\operatorname{Tr}\left[P_{f}^{\alpha_{f}} U_{f, f-1} \cdots P_{2}^{\alpha_{2}} U_{2,1} P_{1}^{\alpha_{1}} \Psi_{0}\right. \\
\left.P_{1}^{\beta_{1}} U_{1,2} P_{2}^{\beta_{2}} \cdots U_{f-1, f} P_{f}^{\beta_{f}}\right],
\end{array}
$$

where $\Psi_{0}$ is some initial state, provided the consistency conditions

$$
\mathbb{D}\left(Y^{\boldsymbol{\alpha}}, Y^{\boldsymbol{\beta}}\right)=0 \text { whenever } \boldsymbol{\alpha} \neq \boldsymbol{\beta}
$$

are satisfied. Here $\boldsymbol{\alpha} \neq \boldsymbol{\beta}$ means that for at least one time $t_{m}$ it is the case that $\alpha_{m} \neq \beta_{m}$. When (19) holds one assigns the positive weight $W(\boldsymbol{\alpha})=$ $\mathbb{D}\left(Y^{\alpha}, Y^{\alpha}\right)$ to the history $Y^{\alpha}$. The probability of each history is its weight divided by the total weight of all the histories; if $\Psi_{0}$ is a normalized density operator this total weight is 1 and the probability of history $\boldsymbol{\alpha}$ is $W(\boldsymbol{\alpha})$.

The Hilbert space for the present discussion is $\mathcal{H}=\mathcal{H}_{M} \otimes \mathcal{H}_{E}$, where $\mathcal{H}_{M}$ is the Hilbert space of the molecule and $\mathcal{H}_{E}$ that of the environment. However, the histories of interest to us refer to properties of the molecule, not the environment, and we employ the usual convention that $P_{m}^{\alpha_{m}}$ representing one of these properties can denote both a projector on $\mathcal{H}_{M}$ or its counterpart $P_{m}^{\alpha_{m}} \otimes I_{E}$ on $\mathcal{H}$. For the initial state we let $\Psi_{0}=I_{M} \otimes\left|\Phi_{E}\right\rangle\left\langle\Phi_{E}\right|$, where

$$
\left|\Phi_{E}\right\rangle=\left|E_{1}\right\rangle \otimes\left|E_{2}\right\rangle \otimes \cdots\left|E_{f-1}\right\rangle
$$

is a "giant" tensor product state on the environment chosen in such a way that during the time interval between $t_{m}$ and $t_{m+1}$ the molecule will interact only with the piece $\left|E_{m}\right\rangle$ in this tensor product in a manner determined by $U_{m+1, m}$; after that this part of the environment can be ignored so far as the molecule is concerned. In particular, if we take a partial trace over the environment of the middle portion on the right side of (18) at time $t_{2}$, the interaction of the 
molecule with $\left|E_{1}\right\rangle$ is chosen so that

$$
\operatorname{Tr}_{E}\left[U_{2,1} P_{1}^{\alpha_{1}} \Psi_{0} P_{1}^{\beta_{1}} U_{1,2}\right]=\mathcal{T}_{2,1}\left(P_{1}^{\alpha_{1}} P_{1}^{\beta_{1}}\right),
$$

where $\mathcal{T}_{2,1}=\mathcal{T}\left(t_{2}-t_{1}\right)$ is the superoperator that maps the state of the molecule at the beginning of this time interval to its state at the end. In the same way, if the partial trace over the environment is carried out at time $t_{3}$ the result will be

$$
\mathcal{T}_{3,2}\left(P_{2}^{\alpha_{2}} \mathcal{T}_{2,1}\left(P_{1}^{\alpha_{1}} P_{1}^{\beta_{1}}\right) P_{2}^{\beta_{2}}\right),
$$

with $\mathcal{T}_{3,2}=\mathcal{T}\left(t_{3}-t_{2}\right)$, and similarly for later times. Consequently, for our model the decoherence functional is given by

$$
\begin{gathered}
\mathbb{D}\left(Y^{\boldsymbol{\alpha}}, Y^{\boldsymbol{\beta}}\right)=\operatorname{Tr}_{M}\left[P _ { f } ^ { \alpha _ { f } } \mathcal { T } _ { f , f - 1 } \left(\cdots P _ { 2 } ^ { \alpha _ { 2 } } \mathcal { T } _ { 2 , 1 } \left(P_{1}^{\alpha_{1}}\right.\right.\right. \\
\left.\left.\left.P_{1}^{\beta_{1}}\right) P_{2}^{\beta_{2}} \cdots\right) P_{f}^{\beta_{f}}\right],
\end{gathered}
$$

an expression which no longer makes any (direct) reference to the environment. See Sec. III of [15] for a more detailed argument.

If all the projectors in the decomposition $\left\{P_{m}^{\alpha_{m}}\right\}$ are rank 1 , which is to say they project onto pure states of the molecule, and the consistency conditions are satisfied, then the probabilities (corresponding to the diagonal elements of the decoherence functional (23)) are those of a memoryless hopping process - a Markov process. If the time steps $t_{m}$ are identical and the same decomposition is used at every time, this process is stationary (homogeneous, i.e. same Markov matrix at each timestep), but in general it is nonstationary (inhomogeneous). Both cases are of interest for our model, as discussed below in Sec. IV.

\section{B. Forwards and backwards conditions}

Finding collections of histories such that the consistency condition (19) is satisfied is made somewhat easier by the following observation. Suppose it is the case that for every $m$ between 1 and $f-1$, if $Q$ is a linear combination of the projectors in the set $\left\{P_{m}^{\alpha_{m}}\right\}$, then $\mathcal{T}_{m+1, m}(Q)$ is a linear combination of the projectors in the set $\left\{P_{m+1}^{\alpha_{m+1}}\right\}$. When this forward condition is satisfied, the family of histories will be consistent, as can be seen in the following way. The functional $\mathbb{D}\left(Y^{\boldsymbol{\alpha}}, Y^{\boldsymbol{\beta}}\right)$ in (23) will vanish if $\alpha_{1} \neq \beta_{1}$, since $P_{1}^{\alpha_{1}} P_{1}^{\beta_{1}}=0$. If the forward condition is satisfied, $\mathcal{T}_{2,1}\left(P_{1}^{\alpha_{1}}\right)$ will be a linear combination of projectors in the collection $\left\{P_{2}^{\alpha_{2}}\right\}$, and will therefore commute with any projector in this collection. Consequently, $P_{2}^{\alpha_{2}} \mathcal{T}_{2,1}\left(P_{1}^{\alpha_{1}}\right) P_{2}^{\beta_{2}}=$ $P_{2}^{\alpha_{2}} P_{2}^{\beta_{2}} \mathcal{T}_{2,1}\left(P_{1}^{\alpha_{1}}\right)$ will vanish whenever $\alpha_{2} \neq \beta_{2}$ since $P_{2}^{\alpha_{2}} P_{2}^{\beta_{2}}=0$, and if it does not vanish it will be some linear combination of the $\left\{P_{2}^{\alpha_{2}}\right\}$. Proceeding in the same way for larger $m$ one sees that $\mathbb{D}\left(Y^{\boldsymbol{\alpha}}, Y^{\boldsymbol{\beta}}\right)$ will vanish if, for any $m, \alpha_{m} \neq \beta_{m}$.

Note that the forward condition is a sufficient but not a necessary condition for consistency. The same is true of the backward condition: for every $m$ between $f$ and 2 it is the case that if $Q$ is a linear combination of the projectors in $\left\{P_{m}^{\alpha_{m}}\right\}$, then $\mathcal{T}_{m, m-1}^{\dagger}(Q)$ is a linear combination of the projectors in $\left\{P_{m-1}^{\alpha_{m-1}}\right\}$. Here $\mathcal{T}_{m, m-1}^{\dagger}$ denotes the adjoint of the superoperator with respect to the Frobenius inner product: $\left\langle\mathcal{T}^{\dagger}(A), B\right\rangle=\langle A, \mathcal{T}(B)\rangle$ where $\langle A, B\rangle=\operatorname{Tr}\left(A^{\dagger} B\right)$. The proof of consistency when the backward condition is satisfied proceeds in the same way as for the forwards condition, but in reverse. Start with (23) and rewrite the argument inside the trace by first cycling $P_{f}^{\beta_{f}}$ to become the first term, and then replacing $\mathcal{T}_{f, f-1}$ with $\mathcal{T}_{f, f-1}^{\dagger}$ acting on $P_{f}^{\beta_{f}} P_{f}^{\alpha_{f}}$, and continue this cycling process to convert all $\mathcal{T}$ to $\mathcal{T}^{\dagger}$. [Note that since $\mathcal{T}$ is a (completely) positive superoperator, $\left(\mathcal{T}^{\dagger}(A)\right)^{\dagger}=\mathcal{T}^{\dagger}\left(A^{\dagger}\right)$.] In the case of qubits, the situation of primary interest for the present paper, one can show that consistent families of histories of the type (17) must satisfy either the forward or the backward condition.

\section{Measuring information}

We will want to discuss and quantify the information about the initial state of the molecule as time goes on. We can do this within the context of the histories formalism. Alternatively, we can do this in the context of the quantum channel formalism, i.e., quantifying the distinguishability of density operators at the output of a quantum channel, and as we will see there is some connection between the two approaches.

Let us first consider information from the histories perspective. Suppose some consistent family of histories uses the projective decompositions $P_{1}=\left\{P_{1}^{j}\right\}$ at time $t_{1}$ and $P_{m}=\left\{P_{m}^{k}\right\}$ at time $t_{m}$, with $t_{1}<t_{m}$ [for simplicity here we replaced the indices $\alpha_{1}$ and $\alpha_{m}$ in (16) and (17) with $j$ and $k$. As in [16], we will equate the notion of a projective decomposition, like $P_{1}$, with a type of information about the system, in our case the molecule. A convenient measure of how much of the $P_{1}$ type of information about the molecule remains at time $t_{m}$ is the Shannon mutual information

$$
H\left(P_{1}: P_{m}\right)=H\left(P_{1}\right)+H\left(P_{m}\right)-H\left(P_{1}, P_{m}\right),
$$

where $H\left(P_{1}\right)$ is the familiar Shannon entropy. In particular if $P_{1}, P_{m}$, and $P_{m^{\prime}}$ are projective decompositions associated with a consistent family at three successive times, and if the probabilities correspond 
to a Markov process, then (see, e.g., p. 510 of 13]) $H\left(P_{1}: P_{m^{\prime}}\right)$ cannot be greater than $H\left(P_{1}: P_{m}\right)$ : the information about the initial situation can only decrease with time. For simplicity, in what follows we will set $\operatorname{Pr}\left(P_{1}^{j}\right)=1 / d_{1}$ for all $j$, where $d_{1}$ is the number of projectors in the decomposition $P_{1}$. Then $H\left(P_{1}: P_{1}\right)=H\left(P_{1}\right)=\log d_{1}$, and hence the information decays from its initial value of $\log d_{1}$ as time goes on.

Now, alternatively, consider the quantum channel perspective, where we will quantify how much of the $P_{1}$ type of information remains at time $t_{m}$ by measuring the distinguishability of the conditional density operators at the output of the relevant quantum channel. (This approach was taken in [12.) To measure distinguishability of density operators, in particular if these density operators do not commute, we need a measure that is inherently quantum-mechanical, which is provided by the Holevo function

$$
\chi\left(\left\{p_{j}, \rho_{j}\right\}\right):=S\left(\sum_{j} p_{j} \rho_{j}\right)-\sum_{j} p_{j} S\left(\rho_{j}\right)
$$

defined for an ensemble $\left\{p_{j}, \rho_{j}\right\}$, where $p_{j}$ is the probability assigned to the density operator $\rho_{j}$, and $S(\rho):=-\operatorname{Tr}(\rho \log \rho)$ is the von Neumann entropy. Applying this measure to the ensemble $\left\{1 / d_{1}, \mathcal{T}_{m, 1}\left(P_{1}^{j}\right)\right\}$, where $\mathcal{T}_{m, 1}$ is the quantum channel that governs the molecule's evolution from $t_{1}$ to $t_{m}$, gives a quantitative measure of how much $P_{1}$ information remains at time $t_{m}$, and we write this as

$$
\hat{\chi}\left(P_{1}, \mathcal{T}(t)\right)=\hat{\chi}\left(P_{1}, \mathcal{T}_{m, 1}\right):=\chi\left(\left\{\frac{1}{d_{1}}, \frac{\mathcal{T}_{m, 1}\left(P_{1}^{j}\right)}{\operatorname{Tr}\left(P_{1}^{j}\right)}\right\}\right),
$$

where $t=t_{m}-t_{1}$.

Equations (24) and (26) give two alternative ways to measure the loss of information from the system over time. Equation (24) has the advantage of a clear conceptual interpretation, whereas Equation (26) has the advantage of being easy to compute since one does not need to go through the histories analysis to compute it. Fortunately, there is a connection between these approaches. It turns out, see the argument in Appendix $\mathrm{D}$ that for a family satisfying the forward consistency condition

$$
H\left(P_{1}: P_{m}\right)=\hat{\chi}\left(P_{1}, \mathcal{T}_{m, 1}\right) .
$$

A similar sort of connection holds for families satisfying the backward consistency condition (but involving the adjoint channel $\mathcal{T}_{m, 1}^{\dagger}$ ), but for simplicity we will focus on families satisfying the forward condition to illustrate information flows in Sect. V

One can also quantify information flow from the molecule to the environment with the quantum channel approach by using the complementary channel with superoperator $\mathcal{T}^{c}$, introduced in Sec. IIA. In fact, $\mathcal{T}^{c}$ is completely determined by $\mathcal{T}$ up to an isometry on its output (the environment), which does not affect distinguishability measures like $\chi$. Hence, the following information measure is welldefined:

$$
\hat{\chi}\left(P_{1}, \mathcal{T}^{c}(t)\right)=\hat{\chi}\left(P_{1}, \mathcal{T}_{m, 1}^{c}\right):=\chi\left(\left\{\frac{1}{d_{1}}, \frac{\mathcal{T}_{m, 1}^{c}\left(P_{1}^{j}\right)}{\operatorname{Tr}\left(P_{1}^{j}\right)}\right\}\right),
$$

where $t=t_{m}-t_{1}$. It quantifies the amount of the $P_{1}$ type of information about the molecule (at time $t_{1}$ ) that is present in the environment at time $t_{m}$. Though one cannot in general equate this $\hat{\chi}\left(P_{1}, \mathcal{T}_{m, 1}^{c}\right)$ with a Shannon mutual information between the molecule and the environment, the former provides, as is well-known (e.g., p. 531 of [13]), an upper bound on the latter.

We note that there can be a tradeoff in sending information to the environment and preserving it in the molecule, which is most dramatic for complementary or mutually-unbiased bases $P_{1}$ and $P_{1}^{\prime}$ :

$$
\hat{\chi}\left(P_{1}, \mathcal{T}_{m, 1}\right)+\hat{\chi}\left(P_{1}^{\prime}, \mathcal{T}_{m, 1}^{c}\right) \leq \log d_{1} .
$$

This inequality is from Corollary 6 of [12].

\section{CONSISTENT FAMILIES FOR OUR MODEL}

\section{A. Differential equations}

Our model has only two states, and therefore any (nontrivial) decomposition of the identity involves only projectors of rank 1 onto pure states. Thus a consistent history family corresponds to a twostate Markov process (sometimes called a "telegraph process"); in general this process is nonstationary: the transition rates depend upon the time. While such a process can be discussed using discrete times separated by finite intervals, the results are simpler and the mathematical expressions more transparent if one adopts a continuous time approximation with differential equations in place of difference equations. It should, of course, be kept in mind that the processes here described are not truly continuous, since time intervals shorter than the correlation time $\tau_{c}$ introduced in Sec. II lack physical significance. The continuous time approach should be satisfactory as long as both $\omega \tau_{c}$ and $\gamma \tau_{c}$ are small compared to 1 . Note that this condition can still be true even when $\gamma$ is large, as long as $\tau_{c}$ decreases as $1 / \gamma$, which seems physically plausible.

For families satisfying the forward consistency condition the relevant differential equations can be obtained in the following way. At a particular time 
the decomposition of the identity will correspond to two projectors, call them $\rho_{0}$ and $\rho_{1}$, represented by end points or antipodes of a diameter of the Bloch sphere. Let the direction of this diameter be denoted by the usual polar and azimuthal angles $\theta$ and $\phi$ : the $z$ axis at $\theta=0$ and the $x$ axis at $\theta=\pi / 2$, $\phi=0$. Which end of the diameter corresponds to these angles does not matter for the following discussion. The locations of these end points after a short time interval is determined by the master equation (13). One can show that because of the form of $\mathbf{S}$ in (17) they are still located on a diameter of the Bloch sphere, but are now a bit closer to its center. The rate of change of the diameter's direction is represented by the differential equations

$$
\frac{d \phi}{d t}=\omega-\gamma \sin 2 \phi, \quad \frac{d \theta}{d t}=\gamma \sin 2 \theta \cos ^{2} \phi,
$$

whereas the shift towards the center can be used to calculate the instantaneous transition rate

$$
\kappa=\gamma\left(1-\sin ^{2} \theta \cos ^{2} \phi\right),
$$

which enters the rate equations

$$
d p_{0} / d t=\kappa\left(-p_{0}+p_{1}\right), \quad d p_{1} / d t=\kappa\left(p_{0}-p_{1}\right)
$$

for the probabilities associated with these two states. The backwards consistency condition can be analyzed in a similar way, and leads to the differential equations

$$
\frac{d \phi}{d t}=\omega+\gamma \sin 2 \phi, \quad \frac{d \theta}{d t}=-\gamma \sin 2 \theta \cos ^{2} \phi .
$$

governing the direction of the diameter, and to exactly the same expression (31) for the transition rate. For a more detailed derivation of these formulas see Appendix B.

\section{B. Stationary families}

If the angles $\theta$ and $\phi$ which determine the diameter for the projectors forming a consistent family do not change with time the Markov process is stationary or homogeneous, in the sense that the states and the transition probabilities do not change with time; of course the actual state of the molecule is varying randomly as it hops back and forth between the two states. The simplest case is what we call the $z$ family, in which $\theta=0$ (or $\pi$ ), thus $d \theta / d t=0$ in (30) or (33) and $d \phi / d t$ is irrelevant. The two projectors $\left(I+\sigma_{z}\right) / 2$ and $\left(I-\sigma_{z}\right) / 2$ correspond, respectively to the even parity (ground) and odd parity (excited) states of the isolated molecule. Thus we have a two-state stationary Markov process in which the molecule spends a certain amount of time in the even parity state before flipping instantaneously (in our continuous time approximation) to the odd parity state where it remains for a random time interval before flipping back. The time $\tau$ between flips is a random variable with an exponential distribution $e^{-\gamma \tau}$, since setting $\theta=0$ in (31) gives

$$
\kappa_{z}=\gamma
$$

for the transition rate. On average the molecule spends an equal amount of time in both states, which means that in our model the environment has an effective temperature $T \gg \hbar \omega / k_{B}$.

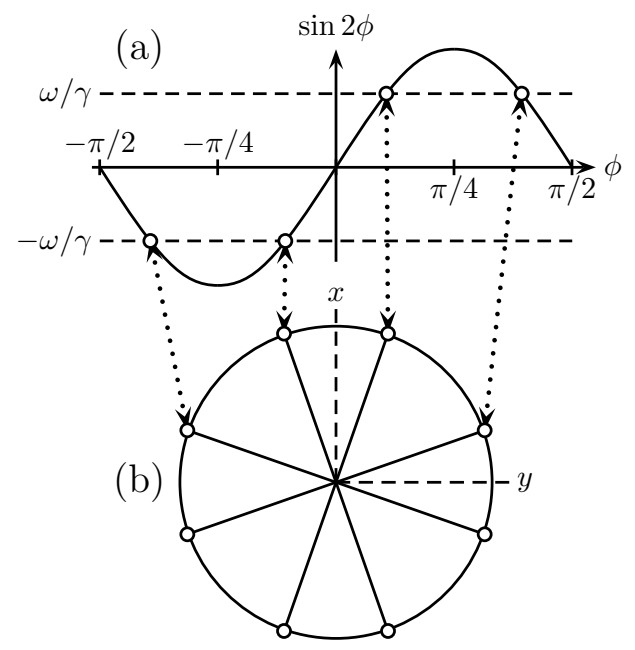

FIG. 1: (a) The steady-state solutions for $\phi$ correspond to the intersections of $\sin 2 \phi$ (solid curve) with $\pm \omega / \gamma$ (dashed lines), shown here for $\gamma>\omega>0$ ("strong decoherence" regime). (b) These steady-state solutions are plotted schematically on the Bloch sphere, as if the $z$ axis is going into the page.

In addition to the $z$ family just discussed there are stationary families in which the projectors correspond to points in the $x-y$ or equatorial plane of the Bloch sphere, so $\theta=\pi / 2$ with $d \phi / d t=0$ in (30) and (33), and thus

$$
\sin 2 \phi= \pm(\omega / \gamma)
$$

For $0<\omega / \gamma<1$ there are four solutions as shown in Fig. 1] which coalesce into two for $\omega / \gamma=1$. For $\omega / \gamma>1$ these families disappear, leaving the $z$ family as the only stationary family. In the limit of strong decoherence, small $\omega / \gamma$, two of the families approach the $x$ axis and two the $y$ axis of the Bloch sphere, so we shall refer to them as the dressed $x$ and dressed $y$-families. The associated transition rates $\kappa_{x}$ and $\kappa_{y}$ are given by $-1 / 2$ times the corresponding eigenvalues of $\mathbf{S}$, see (14):

$\kappa_{x}=-\lambda_{2} / 2=(\gamma-\xi) / 2, \quad \kappa_{y}=-\lambda_{3} / 2=(\gamma+\xi) / 2$.

As $\gamma / \omega$ becomes very large the dressed $x$-families approach the $x$ or chirality basis $|R\rangle$ and $|L\rangle$ of (2), 
and the transition rate $\kappa_{x} \approx \omega^{2} / 4 \gamma$ becomes very slow. Thus these families represent long-lived (almost) chiral states when decoherence is rapid compared with the the tunneling rate. (But see the further discussion in Sec. IVE.)

\section{Nonstationary families}

The equations (30) and (33) can be integrated in closed form to obtain the bases corresponding to nonstationary consistent families, Appendix [C] However, the solutions are fairly complicated expressions. The time evolution for some cases in which $\omega=1$ and $\gamma$ is either less than or larger than 1 is shown in Fig. 2, For $\gamma<\omega$ the diameter rotates continuously about the $z$ axis (the discontinuities in $\phi$ are of course artifacts of the plot) with an angular frequency of

$$
\eta=\sqrt{\omega^{2}-\gamma^{2}},
$$

while the polar angle $\theta$ tends either to $\pi / 2$ for the forward or to 0 (equivalently, $\pi$ ) for the backward consistency condition. In the limit in which $\gamma$ tends to 0 , no decoherence, one has a simple rotation of the diameter of the consistent family about the $z$ axis at a rate $\omega$ with $\theta$ fixed. This same tendency is seen in the dependence of $\theta$ on time when $\gamma>\omega$, whereas $\phi$ more or less rapidly approaches one of the values corresponding to a stationary family. Note that along with the continuous change of basis there is a random flipping from one of the basis states to the other at a rate given by (31), so one is dealing with a nonstationary Markov process.

Thus in the Bloch sphere picture, for $\gamma<\omega$, the families "corkscrew" about the $z$ axis (going away from or towards this axis for the forward or backward families, respectively), with $\phi$ periodically coming back to the same value at time intervals that are integer multiples of $\pi / \eta$. If $\theta=\pi / 2$ it remains constant, so the same basis reoccurs after an interval of $\pi / \eta$. If only these discrete times are considered, the result is what one might call a stroboscopic family which can be thought of as a discrete time stationary Markov process.

\section{Phase transition}

As the parameters $\gamma$ and $\omega$ vary there is a phase transition, a qualitative change of behavior, when they are equal. This manifests itself in a variety of related ways. For $\gamma<\omega$ the eigenvalues of $\mathbf{S}$ include a complex-conjugate pair $\lambda_{2}$ and $\lambda_{3}$, (14), which coalesce into a single degenerate eigenvalue at the transition, and thereafter, for $\gamma>\omega$, become a pair of distinct real eigenvalues. This is, of course, precisely the behavior one finds in a classical one-dimensional oscillator when the damping passes through the critical value. For $\gamma>\omega$ these eigenvalues are the decay rates for the dressed- $x$ and dressed- $y$ continuous stationary Markov processes discussed in Sec. IVB. On the other hand, as $\gamma$ decreases towards $\omega$ from above, the four stationary families shown in Fig. 1(b) coalesce into two, corresponding to diameters of the Bloch sphere midway between the $x$ and $y$ axes, and for $\gamma<\omega$ they no longer exist: the only remaining continuous stationary family is the $z$ family. As noted above in Sec. IVC] for $\gamma<\omega$ there is a new class of "stroboscopic" families defined using a periodic time interval. As $\gamma$ approaches $\omega$ from below this period becomes infinitely long. The behavior of nonstationary continuous families is also different for $\gamma<\omega$ and $\gamma>\omega$. For the former $\phi$ increases indefinitely and monotonically with time, although this motion, which is simply linear when $\gamma=0$, becomes more and more "jerky" as $\gamma$ increases towards $\omega$. See the example for $\gamma / \omega=1 / 2$ in Fig. 2. For $\gamma>\omega, \phi$ approaches a fixed value with increasing time, and no longer "winds." (Again, the damped harmonic oscillator provides a helpful analogy.) One might say that the nonstationary continuous families transition from a damped oscillatory character to a purely damped character as $\gamma / \omega$ increases, passing through the critical value of 1 .

In terms of its mathematical structure as represented in the master equation this is a dynamical quantum phase transition of the sort discussed in quantum optics for two level systems in Ch. 11 of [17 and in [18], and in a more general context in 19 21]. It appears that the vanishing of the inversion transition in ammonia is of this type; see the discussion in Sec. VIB below. We believe that ours is the first attempt to explore dynamical properties near such a transition using the histories approach.

\section{E. Physical interpretation}

Each consistent family contains a collection of histories, and each history a particular succession of micrscopic properties (subspaces of $\mathcal{H}_{M}$ ). One and only one history from this collection will describe the behavior of a particular molecule during a particular interval of time. There is no need to make any reference to measurements, though it is in principle possible (i.e., does not violate the laws of quantum mechanics) to use a succession of suitably idealized measurements to determine which of these histories is actually realized. But because one is dealing with a system exhibiting "quantum" behavior, i.e., in a regime in which a classical description is not adequate, it is important to keep in mind certain respects in which quantum descriptions differ from their classical counterparts. 

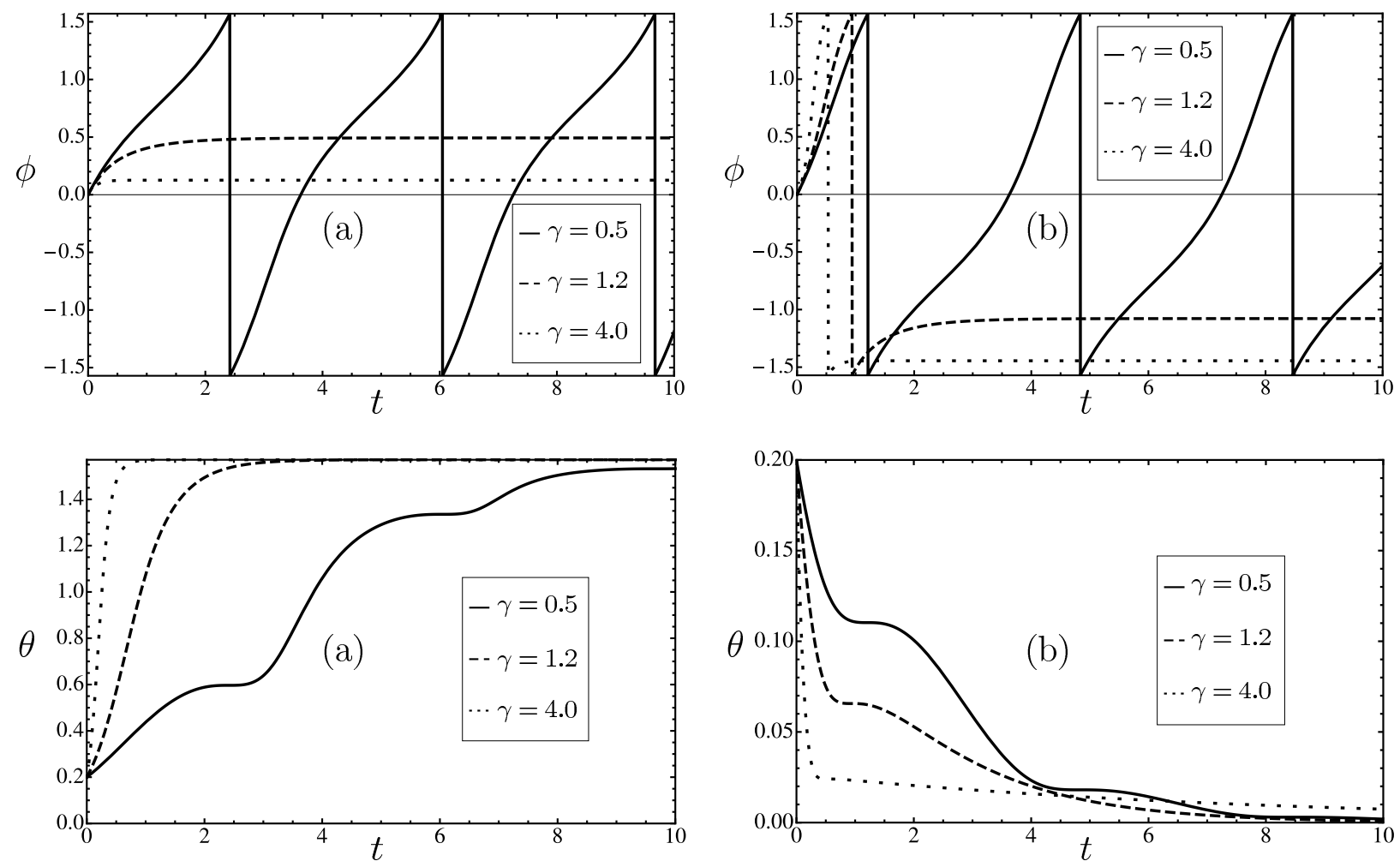

FIG. 2: Time evolution of the Bloch-sphere angles $\phi$ and $\theta$ for the consistent description associated with the (a) forward and (b) backward conditions. In this case, $\phi(0)=0, \theta(0)=0.2, \omega=1$, and $\gamma=0.5,1.2,4$ respectively for the solid, dashed, and dotted curves. The angles $\phi$ and $\theta$ are in units of radians, and the time $t$ is in units of $1 / \omega$.

In particular, two consistent families of histories may be mutually incompatible with each other in such a way that they cannot be combined into a single description that makes sense. A well-known example is a spin-half particle where incompatibility arises from the fact that the operators for angular momentum in different directions do not commute with each other, and hence have no common eigenvectors. It makes good (quantum) sense to say, for example, that $S_{x}=+1 / 2$ (in units of $\hbar$ ), or that $S_{z}=+1 / 2$, but there is no quantum property, no subspace in the Hilbert space, that corresponds to $S_{x}=+1 / 2$ and $S_{z}=+1 / 2$. So one cannot ascribe simultaneous existence to $S_{z}$ and $S_{x}$. In the consistent histories approach this inability to combine incompatible descriptions is codified as the single framework rule, and the consistency conditions discussed above in Sec. III serve to extend this rule from a single time to a sequence of times. In addition, just as two incompatible consistent families or descriptions cannot be combined into a single description, they also cannot be compared: it makes no sense to ask which of two incompatible families is the "correct" one, or to look for some law of nature that single out one against another. Each consistent family provides its own quantum description in a way roughly analogous to looking at a mountain from different locations. For a detailed discussion of these points we refer the reader to [22].

With reference to a tunneling molecule, consider the situation in which $\gamma$ is much larger than $\omega$, strong decoherence. There is a stationary family, Sec.IVB, the "chiral" family, in which the molecule hops back and forth at a comparatively slow rate between the (dressed) left-handed and right-handed chiral states. (There are actually two of these dressed- $x$ families, but when decoherence is strong there is very little difference between them.) This is the family to use if one is interested in understanding why a specific chirality, the left or right-handed form of the molecule, can persist for a very long time in a situation of strong decoherence. It provides a description in terms of a stochastic two-state Markov process in which the rate of hopping from left to right-handed or vice versa is a well-defined function, $\kappa_{x}$ in (36), of the parameters that enter the model. (Each hop is instantaneous on the time scale used for our description, in which intervals less than the correlation time $\tau_{c}$ do not enter; see Sec.【I) As the rate of decoherence decreases, the hopping time becomes shorter and the amount of "dressing" required to produce a consistent family increases, which means that even though this family continues to provide a correct quantum description, it no longer corre- 
sponds to a simple physical picture of a definite leftor right-handed molecule when $\gamma$ becomes comparable to $\omega$.

In addition to this chiral family there is a $z$ or "parity" family in which the molecule hops back and forth at random time intervals between parity eigenstates (energy eigenstates of the isolated molecule), at a rate given by the decoherence rate $\gamma$, see (34). The parity family is incompatible with the chiral family discussed above, and they cannot be combined. One should not try and imagine them as going on simultaneously; to do so would be to make the same mistake as supposing that $S_{x}$ and $S_{z}$ for a spin-half particle can simultaneously possess values. On the other hand, just as it is possible to measure either $S_{x}$ or $S_{z}$, but not both simultaneously, it is also possible in principle (without violating the laws of quantum mechanics) to determine by measurements the succession of events that occur in a parity family, or by a different set of measurements those occurring in a chiral family. Thus a relatively rapid but random flipping back and forth between parity eigenstates is a valid physical picture of the succession of microscopic states of the molecule, one which can be used both when the decoherence is strong and when it is weak. There is in addition a third stationary family for $\gamma>\omega$, the dressed $y$ family, which has a relatively rapid hopping rate in the strong decoherence regime, and eventually merges with the chiral family as the decoherence rate decreases. We do not have a simple name or physical interpretation for this family.

In the regime where decoherence is weak, $\gamma<\omega$, there are no truly stationary families, apart from the parity family discussed above. A relatively simple nonstationary family is the one that employs an "equatorial" basis in the $x-y$ plane of the Block sphere, $\theta=\pi / 2$, rotating at an average angular speed $\eta$, see (37). Let us call this the "tunneling" family, since it corresponds in physical terms to the molecule oscillating back and forth between the two potential wells. As $\gamma$ increases the rate of tunneling decreases and eventually goes to zero at the phase transition $\gamma=\omega$. In addition to the tunneling, the phase $\phi$ undergoes random changes by $\pi$, instantaneous on the time scale we are using, at a rate, (31), proportional to $\gamma$, but also depending on the value of $\phi$. Thus we have a nonstationary Markov process. The random flipping rate increases with $\gamma$ at the same time as the tunneling rate is decreasing, so the simple physical picture of the molecule tunneling from one potential well to the other breaks down upon approaching the phase transition $\gamma=\omega$. For larger values of $\gamma$ this consistent family no longer exists.

\section{INFORMATION FLOWS}

In the previous section we found various consistent frameworks for discussing the stochastic trajectory (for our model) of a tunneling molecule. We now wish to study the dynamics of information, e.g., the loss of information about the molecule's original state as time progresses. Section IIIC discussed how the Shannon mutual information between the original state and the state at some later time, for the forward or backward consistent family, is equivalent to a particular Holevo $\chi$ quantity. Here, for simplicity, we will focus on families satisfying the forward condition, for which the information remaining in the molecule is given by (27), and that flowing to the environment by (28).

These quantities are shown in Fig. 3 for $P_{1}=Z$ (parity basis) and $P_{1}=X$ (chirality basis), both for information remaining in the molecule $\mathcal{T}(t)$ and that flowing to the environment $\mathcal{T}^{c}(t)$. Figure 3(a) shows a case of strong decoherence, $\gamma / \omega=2.5$, where the curves as a function of time are quite smooth, consistent with the fact, Fig. 2(a), that the consistent family is rapidly approaching a stationary family. For weak decoherence, Fig. 3(b) with $\gamma / \omega=0.05$, the consistent family is not stationary and the alternating rises and plateaus reflect this fact. The top curves in both (a) and (b) represent the sums, see (39) below, for one type of information remaining in the molecule and a mutually unbiased type flowing to the environment.

For short times the individual information measures can be computed, using the expressions for $\mathcal{T}$ and $\mathcal{T}^{c}$ in Appendix $\mathrm{A}$ and noting that $S\left(\mathcal{T}^{c}(|\psi\rangle\langle\psi|)\right)=S(\mathcal{T}(|\psi\rangle\langle\psi|))$ for any pure state $|\psi\rangle$, to obtain

$$
\begin{aligned}
\hat{\chi}(X, \mathcal{T}(t)) & =1-O\left[t^{2} \log (1 / t)\right], \\
\hat{\chi}(Z, \mathcal{T}(t)) & =1-\gamma t \log (1 / \gamma t)-\gamma t+O\left[t^{2} \log (1 / t)\right], \\
\hat{\chi}\left(X, \mathcal{T}^{c}(t)\right) & =\gamma t \log (1 / \gamma t)+\gamma t+O\left[t^{2} \log (1 / t)\right], \\
\hat{\chi}\left(Z, \mathcal{T}^{c}(t)\right) & =O\left[t^{2} \log (1 / t)\right],
\end{aligned}
$$

Here $O[$ ] means that the correction term is of this or possibly some higher order. These expressions are consistent with $\hat{\chi}(X, \mathcal{T}(t))$ and $\hat{\chi}\left(Z, \mathcal{T}^{c}(t)\right)$ having zero slope at $t=0$, and $\hat{\chi}(Z, \mathcal{T}(t))$ and $\hat{\chi}\left(X, \mathcal{T}^{c}(t)\right)$ having infinite slope at $t=0$, as depicted in Fig. 3 . That $\hat{\chi}\left(Z, \mathcal{T}^{c}(t)\right)$ in (38) has no term linear in $t$ seems plausible in that the decoherence mechanism in our model has been chosen specifically to carry $X$ information into the environment. The uppermost curves in Figs. 3(a) and 3(b) represent

$$
\begin{aligned}
& \hat{\chi}(Z, \mathcal{T}(t))+\hat{\chi}\left(X, \mathcal{T}^{c}(t)\right)= \\
& \hat{\chi}\left(Z, \mathcal{T}^{c}(t)\right)+\hat{\chi}(X, \mathcal{T}(t))=1-O\left[t^{2} \log (1 / t)\right],
\end{aligned}
$$



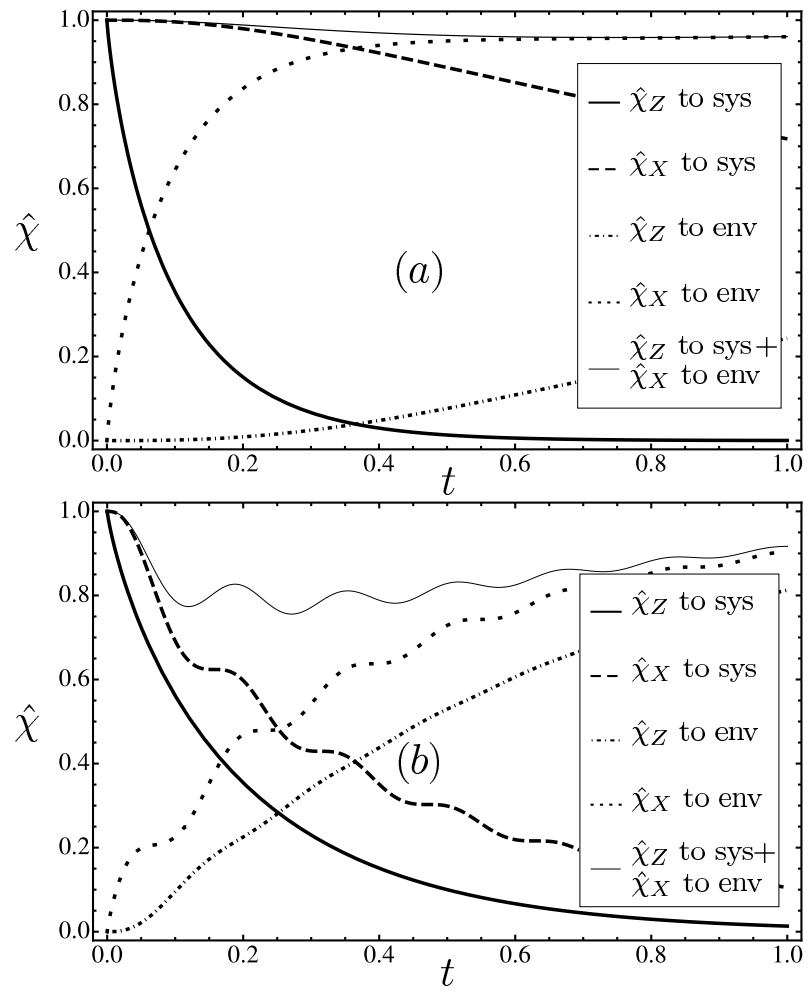

FIG. 3: Information flows in terms of the $\hat{\chi}$ information measure (see text for definition), (a) in the strong decoherence regime with $\gamma=2$ and $\omega=0.8$, and (b) in the weak decoherence regime with $\gamma=1$ and $\omega=20$. The measure $\hat{\chi}$ is in units of bits, and the time $t$ is in units of $0.8 / \omega$ in (a) and $20 / \omega$ in (b).

where the first equality comes from Theorem 3 of [12], and the second from (38); the correction must be negative in view of the bound in (29).

Another consequence of (38) is that for small $t$ the flow of chiral information to the environment is compensated by a decrease of parity information remaining in the molecule:

$$
\frac{\mathrm{d}}{\mathrm{d} t} \hat{\chi}\left(X, \mathcal{T}^{c}(t)\right)=-\frac{\mathrm{d}}{\mathrm{d} t} \hat{\chi}(Z, \mathcal{T}(t))+O[t \log (1 / t)] .
$$

However, as noted above, both sides of (40) diverge logarithmically as $t \rightarrow 0$. Of course, these expressions lack physical meaning for times shorter than $\tau_{c}$, and thus the divergence is a mathematical artifact. Nonetheless, this makes it difficult to define rates of flow of information in a mathematically clean way using the $\hat{\chi}$ measure.

An alternative which avoids the divergence is to replace the von Neumann entropy $S$ in the definition (25) with the quadratic entropy

$$
S_{Q}(\rho)=1-\operatorname{Tr}\left(\rho^{2}\right)
$$

In the case of a qubit channel with $W$ an orthonormal basis, projectors $W^{1}+W^{2}=I$, and Pauli oper- ator $\sigma_{W}:=W^{1}-W^{2}$, the measure defined in (26) becomes

$$
\hat{\chi}_{Q}(W, \mathcal{T}(t))=\frac{1}{2} \operatorname{Tr}\left[\left(\mathcal{T}\left(\sigma_{W}\right)\right)^{2}\right],
$$

with a similar expression for the complementary channel if $\mathcal{T}$ is replaced by $\mathcal{T}^{c}$. One can use the usual Pauli representation to write

$$
\sigma_{W}=\boldsymbol{n} \cdot \boldsymbol{\sigma},
$$

where the $x, y$, and $z$ components of the $\sigma_{W}$ Pauli operator are given by the unit vector

$$
\boldsymbol{n}=\left\{n_{x}, n_{y}, n_{z}\right\}=\{\sin \theta \cos \phi, \sin \theta \sin \phi, \cos \theta\} .
$$

Using the short-time expressions for $\mathcal{T}$ and $\mathcal{T}^{c}$ given in Appendix $\mathrm{A}$ one finds that for the direct and complementary channels

$$
\begin{aligned}
\hat{\chi}_{Q}(W, \mathcal{T}(t)) & =1-4 \gamma t\left[1-n_{x}^{2}\right]+O\left(t^{2}\right), \\
\hat{\chi}_{Q}\left(W, \mathcal{T}^{c}(t)\right) & =4 \gamma t n_{x}^{2}+O\left(t^{2}\right) .
\end{aligned}
$$

Setting $W=X$ in (46) and $W=V$ in (45), where $V$ is some basis in the $y-z$ plane and thus mutually unbiased relative to $X$, the analog of (40) with $\hat{\chi}$ replaced by $\hat{\chi}_{Q}$ is

$$
\frac{d}{d t} \hat{\chi}_{Q}\left(X, \mathcal{T}^{c}(t)\right)=-\frac{d}{d t} \hat{\chi}_{Q}(V, \mathcal{T}(t))=4 \gamma
$$

at $t=0$, so the derivatives are now finite. Thus when one uses the $\hat{\chi}_{Q}$ measure the rate of flow of $X$ information to the environment equals the rate of decrease within the molecule of any type of information associated with a basis in the $y$ - $z$ plane.

\section{VI. $\mathbf{D}_{2} \mathrm{~S}_{2}$ AND $\mathrm{NH}_{3}$}

\section{A. $\mathbf{D}_{2} \mathbf{S}_{2}$}

An order-of-magnitude calculation of the decoherence rate $\gamma$ for a $\mathrm{D}_{2} \mathrm{~S}_{2}$ molecule immersed in a gas of helium atoms shows that this system is in the strong decoherence regime under typical conditions. The flux $q$ of helium atoms (atoms per unit area per unit time) is their concentration times their average velocity, given by $q=P \cdot \sqrt{8 /\left(\pi m_{\mathrm{He}} k_{b} T\right)}$, assuming an ideal gas with pressure $P$ and temperature $T$, with $m_{\mathrm{He}}$ and $k_{b}$ the mass of a helium atom and Boltzmann's constant 23]. At room temperature $T=300 \mathrm{~K}$ and a pressure $P$ of 1 atmosphere this gives $q \approx 3 \times 10^{28}$ atoms $\mathrm{s}^{-1} \mathrm{~m}^{-2}$. Multiplying $q$ by approximate cross sections $\sigma_{\text {col }} \approx 1000 a_{0}^{2}$ and $\sigma_{\mathrm{dec}} \approx 100 a_{0}^{2}$ for collisions and decoherence, taken from [7], with $a_{0}$ the Bohr radius, leads to a collision 
rate of approximately $9 \times 10^{10} s^{-1}$ and a decoherence rate of

$$
\gamma \approx 9 \times 10^{9} \mathrm{~s}^{-1} .
$$

The estimated tunneling rate is $\omega \approx 176 \mathrm{rad} / \mathrm{s}$ after correcting $^{2}$ the value published in [7] by a factor of $2 \pi$. Thus

$$
\gamma / \omega \approx 5 \times 10^{7},
$$

which means strong decoherence, for which our results in Sec. IV indicate that chirality is both a consistent description as well as a stable property, to a very good approximation. Probing the regime $\gamma \leq \omega$ for this molecule would seem quite difficult as it would involve very low pressures. Replacing deuterium with hydrogen and/or sulfur with oxygen leads to chiral molecules, e.g. $\mathrm{H}_{2} \mathrm{O}_{2}$, that have significantly higher tunneling frequencies [24] and hence may be candidates for probing the $\gamma \leq \omega$ regime.

\section{B. $\mathbf{N H}_{3}$}

In the electronic ground state of ammonia $\mathrm{NH}_{3}$ the nitrogen lies to one side of the plane defined by the three hydrogens, but there is a relatively low potential barrier separating it from the mirror image state on the other side, and tunneling in this double-well potential exhibits itself in the well-known inversion transition at a frequency of about $24 \mathrm{GHz}$. Although the molecule is not chiral, when it is rotating about an axis passing through the nitrogen and the midpoint between the hydrogen atoms the symmetry operation of parity (inversion) moves the nitrogen to the other side of the plane, changing the sign of the electric dipole, while leaving the angular momentum unchanged. Consequently, the energy levels with a nonzero quantum number $K$ for this component of angular momentum are split into two parity eigenstates by the inversion transition in a way similar to that in a chiral molecule.

The tunneling transition has been observed directly by microwave absorption, which at low pressure exhibits a set of closely-spaced lines associated with the different rotational states [25]. As the pressure increases the lines broaden and merge, and the center of the merged line shifts towards lower frequencies, reaching zero frequency at a pressure of about 2 atmospheres [26]. It has been suggested, e.g. [27], that at pressures above this transition the ammonia molecule adopts a "pyramidal" shape with the nitrogen on one side of the hydrogen plane, analogous to the shape of a chiral molecule with a definite handedness. Deuterated ammonia $\mathrm{ND}_{3}$ shows

\footnotetext{
${ }^{2}$ Private communication from K. Hornberger
}

similar behavior, except that the low pressure tunneling frequency is now at $1.6 \mathrm{GHz}$, and the center of the broadened line tends to zero frequency at a pressure of 0.12 atmospheres [28].

The shift towards zero frequency has been analyzed theoretically using two different approaches. The first, exemplified by [29], and with similar ideas in [30, 31] among others, starts with the observation that since the ammonia molecule possesses a significant electric dipole moment when the nitrogen is on one side of the hydrogen plane there will be a strong dipole-dipole interaction between nearby molecules. It is then proposed that this produces a sort of mean-field effect in which the polarization of one molecule influences its neighbors in such a way that eventually as the pressure increases and the molecules come closer together, the double well potential for a single molecule is changed into one with a single minimum on one side of the hydrogen plane, resulting in molecules of pyramidal shape.

An alternative approach found in 32 35 focuses instead on the decohering effects of collisions between gas molecules. It is argued that these collisions in addition to broadening the lines can also lower the tunneling frequency as the pressure, and thus the collision rate, increases. From this perspective the electric dipole-dipole interaction, while significant in determining the collision cross section and the effects of collisions, is not the fundamental source of the line shift to lower frequencies. The latter ought still to be present if ammonia is a dilute component in a nonpolar buffer gas. Of particular significance for this second point of view is the work of Ben-Reuven 32, 33], who argued on theoretical grounds that when proper account is taken of the effects of collisions the line shape, absorption as a function of frequency, is not adequately represented by the Van Vleck and Weisskopf formula [36] used earlier in [26] to analyze the experimental data. He proposed an alternative line shape function with three parameters, $\gamma, \zeta$ and $\delta$, proportional to the collision rate, and thus the pressure, to fit the experimental microwave absorption data for $\mathrm{NH}_{3}$ and (with a different choice of parameters) $\mathrm{ND}_{3}$ over a range of frequencies and pressures sufficient to include that at which the tunneling frequency goes to zero. It is noteworth that this fit was achieved for all pressures and frequencies using just these three parameters, whereas the earlier analysis of Bleaney and Loubser [26] was carried out by adjusting two parameters separately for each pressure.

The validity of Ben-Reuven's analysis is supported by the fact that more recent data on microwave absorption by ammonia in mixtures of hydrogen and helium (of interest in studies of the atmospheres of Jupiter and the other giant planets) has been fitted using his line shape formula for the tunneling transition [37] with, of course, different choices of parame- 
ters for the different species scattering from the ammonia molecule. Since neither hydrogen nor helium has an electric dipole moment, this tends to support the idea that collisions, rather than dipole-dipole interactions as such, are what drive the transtion frequency to zero in pure ammonia gas as the pressure rises. The numbers given in [37] would suggest a phase transition at about 20 atmospheres for ammonia in a buffer gas of hydrogen at room temperature. Replacing $\mathrm{NH}_{3}$ with $\mathrm{ND}_{3}$ should bring the transition pressure down by a factor of 15 , and replacing hydrogen with a gas of some other nonpolar molecule might be advantageous. Thus a direct experimental test of whether dipole-dipole interactions are or are not essential for understanding the vanishing of the tunneling frequency seems feasible.

Our very simple decoherence model corresponds to setting $\gamma=\zeta$ and $\delta=0$ in Ben-Reuven's theory as it applies to a two-level system. In fact, he achieved a good fit to the experimental data with $\delta=0$, but with $\gamma$ larger than $\zeta$ by a factor of around 1.3, see p. 21 of [33]. To have $\gamma$ larger than $\zeta$ in our model would require our adding another source of decoherence. The phase transition present in our model is also clearly present in Ben-Reuven's work; see the discussion of the spectrum of the perturbed Liouville matrix in Sec. $4 \mathrm{C}$ of [33], where the eigenvalues change character when $\zeta$ passes through the value $\omega_{0}+\delta$; this is the counterpart of our $\gamma=\omega$. Hence it seems that the vanishing of the inversion frequency in ammonia with increasing pressure is an instance of the sort of phase transition that occurs in our model. A more detailed comparison, which we have not attempted, would require our including an additional mechanism for decoherence to make $\gamma$ larger than $\zeta$, and dealing with complications caused by the presence in ammonia of a number of different rotational states. Nonetheless, we think our considerations provide some insight into the sense in which the ammonia molecule can be said to be "pyramidal" in the gas at high pressure and lack this feature at low pressures. Namely, when collisions are sufficiently frequent there is a consistent family of histories, the chiral or $x$ family discussed in Sec. IVE, in which in quantum mechanical terms the molecule spends a time much longer than the tunneling time in a pyramidal shape (or a "dressed" state close to it) which is "chiral" in the sense that electric dipole moment has a definite orientation relative to the angular momentum, with occasional random hops between the two pyramidal possibilities. As the pressure decreases towards the transition pressure the pyramidal picture begins to break down: the hops become more frequent between dressed states, which are starting to lose their pyramidal character. At still lower pressures it is better to think of the molecule as continuously tunneling back and forth, rather than possessing a fixed pyramidal form, with a period that diverges as the pressure rises to its value at the transition.

\section{CONCLUSION}

We have shown how the decoherence of a two-state tunneling molecule, a chiral molecule or ammonia, in the presence of a buffer gas can be described in terms of a succession of quantum states of the molecule itself that form a consistent family of histories, on a sufficiently coarse time scale so that intervals are always longer than a correlation time. Our model is described by just two parameters, a tunneling rate $\omega$ and a decoherence rate $\gamma$, and its essential properties depend upon the ratio $\gamma / \omega$. In addition we have studied the flow of information to the environment, along with its retention by the molecule itself, during the process of decoherence.

We found a large variety of consistent families, some of which are stationary (in the sense of Markov processes) and some of which are not. In the regime $\gamma / \omega \gg 1$ of strong decoherence there is a stationary family (actually two closely related families) in which the molecule spends a relatively long time in one of its chiral states before flipping to the other chirality, and eventually flipping back again, in a stationary Markov ("telegraph") process, with a transition rate which is approximately $\omega^{2} / 4 \gamma$ for $\gamma \gg \omega$, and hence quite slow compared to the tunneling rate $\omega$. Thus this "chiral" family explains the persistence of chirality for a long period of time when there is strong decoherence. However, as $\gamma / \omega$ decreases, the transitions between chiral states become more frequent and the states themselves (the "dressed $x$ " states of Sec. IVB) lose their chiral character, until finally this family disappears entirely at a phase transition $\gamma / \omega=1$.

We have found two other stationary consistent families for $\gamma / \omega>1$. One of them is the "parity" family in which the molecule is at each of the times considered in one of the two states of definite parity (the energy eigenstates of the isolated molecule), but with a transition rate of $\gamma$ between them, thus a rapid flipping compared to transitions between chiral states when the decoherence is large. This family, present at all values of $\gamma / \omega$, is incompatible, in the quantum mechanical sense, with the chiral family: while both provide valid quantum descriptions, they cannot be employed simultaneously; see the discussion in Sec. IVE The other stationary family for $\gamma / \omega>1$ (again there are actually two families) is the "dressed $y$ " family of Sec. IVB It involves a relatively rapid flipping between two orthogonal quantum states for which we do not have a simple physical interpretation. Like the chiral family this one only exists for $\gamma>\omega$.

For $\gamma<\omega$ there is a nonstationary "tunneling" 
family in which the molecule oscillates back and forth between the two chiral states at a rate that goes to zero as $\gamma / \omega$ increases to 1 . On top of this relatively smooth oscillation there are random changes in phase which constitute a nonstationary Markov process, with a rate that increases with $\gamma$. This tunneling family disappears at the phase transition $\gamma=\omega$. The only truly stationary family in the regime $\gamma<\omega$ is the parity family. In addition, both for $\gamma<\omega$ and for $\gamma>\omega$ there are a variety of nonstationary consistent families in which the orthogonal basis used to describe the quantum system tends with time towards one of the stationary families or, for $\gamma<\omega$, the tunneling family.

It seems likely that most chiral molecules under most conditions will be in a regime of strong decoherence $\gamma \gg \omega$, see the remarks about $\mathrm{D}_{2} \mathrm{~S}_{2}$ in Sec. VIA Whereas it can only be thought of as "chiral" when in an appropriate rotational state, ammonia, including its deuterated form $\mathrm{ND}_{3}$, is an example of a tunneling molecule in which the transition at $\gamma / \omega=1$ can be readily observed in the laboratory. Indeed, it appears that it has already been observed; see the discussion in Sec. VIB, One wonders if additional experiments, perhaps using technques other than, or in addition to, microwave absorption might be helpful in elucidating its behavior near the phase transtion.

In addition to consistent quantum families of histories we have studied, within the scope of our simple model, the flow of information from a tunneling molecule to its environment, along with the loss of information in the molecule itself. In Sec. $\mathrm{V}]$ we used a perspective in which at a later time the information about the quantum state of the molecule at an earlier time is thought of as a quantum channel, while similar information present at this later time in the environment constitutes a complementary channel. What happens in both cases depends strongly on the type of information considered. Given that our model of decoherence, Sec. IIB, is based on the flow of chiral $(X)$ information - is the molecule left or right handed? - to the environment, we were not surprised to find this exhibited in our quantitative measures, together with a rapid decrease of "complementary" types of information, corresponding to bases mutually unbiased with respect to $X$, retained within the molecule itself. Indeed, there is a direct quantitative relationship for short times if one uses a Holevo type of information measure, and an exact equality in the instantaneous rates, (47), if in the Holevo measure von Neumann entropy is replaced with quadratic entropy in order to render the rates finite.

There are a number of ways in which lines of investigation initiated in this paper could be further extended. Parity-violation effects could be modeled as a small energy splitting between chiral states
38]. Also, our model contains only one mechanism for decoherence, Sec. II] adding a second would allow a serious comparison with Ben-Reuven's formula for ammonia as discussed in Sec. VIB Obtaining the correct physical interpretation might prove difficult given the complexity of the rotational states, even for ammonia present as a dilute component in a buffer gas. Fluorescence from a two-level atom, where decoherence arises from spontaneous decay, could be a simpler system for studying the dynamical phase transition. This transition has been studied in terms of correlations among scattered photons in [18], and it would be of interest to supplement this with a description of how the atom itself behaves as a function of time. Indeed, even the decay of an isolated atom initially in an excited state has not, so far as we know, been examined using consistent families, and studying them might yield valuable physical insights.

\section{Acknowledgments}

The research described here was supported by the Office of Naval Research. V. Gheorghiu acknowledges additional support from the Natural Sciences and Engineering Research Council of Canada (NSERC) and from a Pacific Institute for Mathematical Sciences (PIMS) postdoctoral fellowship.

\section{Appendix A: Explicit expressions for $\mathrm{T}$ and its eigenvectors}

The general expression for $\mathbf{T}(t)=e^{t \mathbf{S}}$ in (7) is

$$
\mathbf{T}(t)=\left(\begin{array}{cccc}
1 & 0 & 0 & 0 \\
0 & e^{-\gamma t} a(t) & -e^{-\gamma t} b(t) & 0 \\
0 & e^{-\gamma t} b(t) & -e^{-\gamma t} c(t) & 0 \\
0 & 0 & 0 & e^{-2 \gamma t}
\end{array}\right)
$$

where, for $\gamma>\omega$

$$
\begin{aligned}
a(t) & =\cosh \xi t+(\gamma / \xi) \sinh \xi t \\
b(t) & =(\omega / \xi) \sinh \xi t \\
c(t) & =\cosh \xi t-(\gamma / \xi) \sinh \xi t \\
\xi & :=\sqrt{\gamma^{2}-\omega^{2}}
\end{aligned}
$$

whereas for $\gamma<\omega$

$$
\begin{aligned}
a(t) & =\cos \eta t+(\gamma / \eta) \sin \eta t \\
b(t) & =(\omega / \eta) \sin \eta t \\
c(t) & =\cos \eta t-(\gamma / \eta) \sin \eta t \\
\eta & :=\sqrt{\omega^{2}-\gamma^{2}} .
\end{aligned}
$$

For $\gamma=\omega$ one has

$$
a(t)=1+\gamma t, \quad b(t)=\gamma t, \quad c(t)=1-\gamma t,
$$


where of course $\gamma$ could be replaced by $\omega$.

For the eigenvalues $\lambda_{1}$ and $\lambda_{4}$ in (14) the left and right eigenvectors of $\mathbf{T}$ are, trivially, $(1,0,0,0)$ and $(0,0,0,1)$, respectively. For $\lambda_{2}$ and $\lambda_{3}$, the unnormalized left $\boldsymbol{v}$ and (transposed) right $\boldsymbol{w}$ eigenvectors are:

$$
\begin{array}{ll}
\boldsymbol{v}_{2}=(0, \xi-\gamma, \omega, 0), & \boldsymbol{w}_{2}=(0, \gamma-\xi, \omega, 0) \\
\boldsymbol{v}_{3}=(0,-\gamma-\xi, \omega, 0), & \boldsymbol{w}_{3}=(0, \gamma+\xi, \omega, 0),
\end{array}
$$

where for $\gamma<\omega$ replace $\xi$ with $i \eta$.

For short times $t \ll 1$ one has

$$
\mathbf{T}(t)=\left(\begin{array}{cccc}
1 & 0 & 0 & 0 \\
0 & 1 & -\omega t & 0 \\
0 & \omega t & 1-2 \gamma t & 0 \\
0 & 0 & 0 & 1-2 \gamma t
\end{array}\right)+\mathbf{O}\left(t^{2}\right)
$$

for the direct channel and

$$
\begin{aligned}
\mathbf{T}^{c}(t)= & \left(\begin{array}{cccc}
1 & 0 & 0 & 0 \\
0 & 2 \sqrt{\gamma} \sqrt{t}-\gamma^{3 / 2} t^{3 / 2} & \omega \sqrt{\gamma} t^{3 / 2} & 0 \\
0 & 0 & 0 & 0 \\
1-2 \gamma t & 0 & 0 & 0
\end{array}\right) \\
& +\mathbf{O}\left(t^{2}\right) .
\end{aligned}
$$

for the complementary channel. During this short time interval, one can represent the direct channel $\mathcal{T}$ using using only 2 Kraus operators, instead of 4 required by the most general qubit channel. The physical intuition behind this is that during this time interval the system of interest interacts only with a qubit environment, being effectively "decoupled" from the other environmental qubit (we remind the reader that the most general qubit evolution requires an interaction with an environment that is represented by at least 2 qubits, see e.g. [13]).

\section{Appendix B: Differential equations for consistent families}

As discussed in Sec. IV A the forwards and backwards consistency conditions specify how a diameter of the Bloch sphere rotates. To determine this for the forwards condition, consider the density operator which at the initial time is at one end of the diameter, and write it in the form

$$
\rho=\frac{1}{2}(I+\boldsymbol{r} \cdot \boldsymbol{\sigma}), \quad \boldsymbol{r}=r \boldsymbol{n},
$$

using the notation of (43) and (44). Because $\mathcal{T}$ is unital the master equation (12) for $\rho$ is equivalent to

$$
d \boldsymbol{r} / d t=\overline{\mathbf{S}} \cdot \boldsymbol{r}
$$

or

$$
\left(\frac{d r}{d t}\right) \boldsymbol{n}+\left[r \frac{d \boldsymbol{n}}{d t}\right]=(\boldsymbol{n} \cdot \overline{\mathbf{S}} \cdot \boldsymbol{n}) \boldsymbol{n}+[\overline{\mathbf{S}} \cdot \boldsymbol{r}-(\boldsymbol{n} \cdot \overline{\mathbf{S}} \cdot \boldsymbol{n}) \boldsymbol{n}]
$$

where $\overline{\mathbf{S}}$ is the lower right $3 \times 3$ block of $\mathbf{S}$ in (7). Set $r=1$, thus $\boldsymbol{r}=\boldsymbol{n}$, and take the dot product of both sides of (B3) with $\boldsymbol{n}$, noting that $\boldsymbol{n}$ and $d \boldsymbol{n} / d t$ are necessarily orthogonal to each other, to obtain:

$$
\begin{aligned}
d r / d t & =\boldsymbol{n} \cdot \overline{\mathbf{S}} \cdot \boldsymbol{n}, \\
d \boldsymbol{n} / d t & =\overline{\mathbf{S}} \cdot \boldsymbol{n}-(\boldsymbol{n} \cdot \overline{\mathbf{S}} \cdot \boldsymbol{n}) \boldsymbol{n} .
\end{aligned}
$$

Note that $\boldsymbol{n} \cdot \overline{\mathbf{S}} \cdot \boldsymbol{n}$ depends only on the symmetrical part of $\overline{\mathbf{S}}$, which is to say the dissipative term, proportional to $\gamma$, in the master equation (13). The differential equations in (30) are equivalent to (B5) when $\boldsymbol{n}$ is written in polar coordinates. To obtain the differential equations for the backwards consistency condition, replace $\overline{\mathbf{S}}$ in (B5) with $\overline{\mathbf{S}}^{\dagger}$, corresponding to the adjoint superoperator $\mathcal{T}^{\dagger}$, and $d / d t$ with $-d / d t$. The resulting differential equations are equivalent to (30) with $\gamma$ replaced with $-\gamma$, thus (33).

The consistency conditions are related to the motion in the Bloch sphere of the diameter that corresponds to the (instaneous) orthonormal basis. However, the instantaneous hopping rate $\kappa$ for the Markov process can be calculated using the Born rule for a very short time interval during which one can assume that the diameter remains fixed, as its motion (the change in basis) only contributes to higher order. When $r=1, \kappa$ as defined in (32) is equal to $(-1 / 2) d r / d t$. Thus, using (B-B),

$$
\kappa=(-1 / 2)(\boldsymbol{n} \cdot \overline{\mathbf{S}} \cdot \boldsymbol{n})=\gamma\left(1-n_{x}^{2}\right),
$$

which, transformed to polar coordinates, is (31). 


\section{Appendix C: Time-integrated solutions for consistent families}

It is helpful to define $\mu(t):=\tan \phi(t)$ and $\nu(t):=\tan \theta(t)$. Then (30) and (33) can be integrated to give the following explicit solutions for the non-stationary consistent families:

$$
\begin{aligned}
& \mu(t)=\mu(0)+\left(\omega \mp 2 \gamma \mu(0)+\omega \mu(0)^{2}\right) \frac{\sinh \xi t}{\xi \cosh \xi t+( \pm \gamma-\omega \mu(0)) \sinh \xi t} \\
& \nu(t)=\nu(0) e^{ \pm \gamma t} \sqrt{1 \pm(\gamma / \xi) \frac{1-\mu(0)^{2}}{1+\mu(0)^{2}} \sinh 2 \xi t+\left(2 \gamma / \xi^{2}\right)\left(\gamma \mp \frac{2 \omega \mu(0)}{1+\mu(0)^{2}}\right) \sinh ^{2} \xi t}
\end{aligned}
$$

where the top (or bottom) symbol in \pm or $\mp$ is the solution to (30) (or (33)) for the family satisfying the forward (or backward) condition. In the case that $\omega>\gamma$, one can replace every occurrence of $\xi=\sqrt{\gamma^{2}-\omega^{2}}$ in (C1) with $\eta=\sqrt{\omega^{2}-\gamma^{2}}$, provided that sinh and cosh are replaced by sin and cos.

\section{Appendix D: Equality of mutual information and $\chi$ measure when forwards consistency conditions satisfied}

The key observation is that, when the forward condition is satisfied, $\mathcal{T}_{m, 1}\left(P_{1}^{j}\right)=\sum_{k} q_{k j} P_{m}^{k}$ for each $j$ and hence the ensemble of density operators at the channel output commute with each other, so quantum (von Neumann) entropies of these density operators become classical (Shannon) entropies in the basis that diagonalizes these density operators. Denoting $r_{1}^{j}:=\operatorname{Tr}\left(P_{1}^{j}\right)$ and $r_{m}^{k}:=\operatorname{Tr}\left(P_{m}^{k}\right)$, we have

$$
\begin{aligned}
\hat{\chi}\left(P_{1}, \mathcal{T}_{m, 1}\right)= & S\left(\sum_{j} p_{j} \mathcal{T}_{m, 1}\left(P_{1}^{j}\right) / r_{1}^{j}\right)-\sum_{j} p_{j} S\left(\mathcal{T}_{m, 1}\left(P_{1}^{j}\right) / r_{1}^{j}\right) \\
= & S\left(\sum_{j, k} p_{j} q_{k j} P_{m}^{k} / r_{1}^{j}\right)-\sum_{j} p_{j} S\left(\sum_{k} q_{k j} P_{m}^{k} / r_{1}^{j}\right) \\
= & H\left(\left\{\sum_{j} p_{j} q_{k j} r_{m}^{k} / r_{1}^{j}\right\}_{k}\right)+\sum_{j, k}\left(p_{j} q_{k j} r_{m}^{k} / r_{1}^{j}\right) S\left(P_{m}^{k} / r_{m}^{k}\right) \\
& -\sum_{j} p_{j} H\left(\left\{q_{k j} r_{m}^{k} / r_{1}^{j}\right\}_{k}\right)-\sum_{j, k}\left(p_{j} q_{k j} r_{m}^{k} / r_{1}^{j}\right) S\left(P_{m}^{k} / r_{m}^{k}\right) \\
= & H\left(\left\{\sum_{j} p_{j} q_{k j} r_{m}^{k} / r_{1}^{j}\right\}_{k}\right)-\sum_{j} p_{j} H\left(\left\{q_{k j} r_{m}^{k} / r_{1}^{j}\right\}_{k}\right) \\
= & H\left(P_{m}\right)-H\left(P_{m} \mid P_{1}\right)=H\left(P_{1}: P_{m}\right),
\end{aligned}
$$

where the $k$ subscript in $\{\cdot\}_{k}$ indicates that the set is generated by allowing $k$ to vary. In this derivation, we used a property of the von Neumann entropy, for orthogonal density operators, given on page 513 of 13 .

[1] M. B. Plenio and P. L. Knight. The quantum-jump approach to dissipative dynamics in quantum optics. Rev. Mod. Phys., 70:101-144, 1998.

[2] Todd A. Brun. A simple model of quantum trajectories. Am. J. Phys., 70:719-737, 2002. quant-ph/0108132.

[3] Todd A. Brun. Quantum jumps as decoherent histories. Phys. Rev. Lett., 78:1833-1837, Mar 1997.

[4] F. Hund. Zur Deutung der Molekelspektren. III. Zeitschrift für Physik, 43:805-826, 1927.

[5] Markus Simonius. Spontaneous symmetry breaking and blocking of metastable states. Phys. Rev. Lett.,
40(15):980-983, Apr 1978.

[6] B. R. Fischer and P. Mittelstaedt. Chirality as a quasi-classical property of molecular systems. Physics Letters A, 147(8-9):411-416, 1990.

[7] Johannes Trost and Klaus Hornberger. Hund's paradox and the collisional stabilization of chiral molecules. Phys. Rev. Lett., 103(2):023202, Jul 2009.

[8] E. Joos, H. D. Zeh, C. Kiefer, D. Giulini, J. Kupsch, and I.-O. Stamatescu. Decoherence and the Appearance of a Classical World in Quantum Theory. Springer-Verlag, Berlin, 2d edition, 2003. 
[9] Wojciech Hubert Zurek. Decoherence, einselection, and the quantum origins of the classical. Rev. Mod. Phys., 75:715-1083, 2003.

[10] K. Hornberger. Monitoring approach to open quantum dynamics using scattering theory. EPL (Europhysics Letters), 77(5):50007, 2007.

[11] Amnon Aharony, Shmuel Gurvitz, Ora EntinWohlman, and Sushanta Dattagupta. Retrieving qubit information despite decoherence. Phys. Rev. B, 82:245417, Dec 2010.

[12] Patrick J. Coles, Li Yu, Vlad Gheorghiu, and Robert B. Griffiths. Information-theoretic treatment of tripartite systems and quantum channels. Phys. Rev. A, 83(6):062338, 2011.

[13] Michael A. Nielsen and Isaac L. Chuang. Quantum Computation and Quantum Information. Cambridge University Press, Cambridge, 5th edition, 2000.

[14] Robert B. Griffiths. Consistent Quantum Theory. Cambridge University Press, Cambridge, 2002.

[15] Juan Pablo Paz and Wojciech Hubert Zurek. Environment-induced decoherence, classicality, and consistency of quantum histories. Phys. Rev. D, 48(6):2728-2738, Sep 1993.

[16] Robert B. Griffiths. Types of quantum information. Phys. Rev. A, 76(6):062320, 2007.

[17] D. F. Walls and G. J. Milburn. Quantum Optics. Springer-Verlag, Berlin, 1994.

[18] Juan P. Garrahan and Igor Lesanovsky. Thermodynamics of quantum jump trajectories. Phys. Rev. Lett., 104:160601, 2010.

[19] Horacio M. Pastawski. Revisiting the Fermi Golden Rule: Quantum dynamical phase transition as a paradigm shift. Physica B, 398:278-286, 2007.

[20] Ingrid Rotter. A non-Hermitian Hamilton operator and the physics of open quantum systems. J. Phys. A, 42:153001, 2009.

[21] Ingrid Rotter. Dynamical phase transitions in quantum systems. J. Modern Phys., 1:303-311, 2010.

[22] Robert B. Griffiths. A consistent quantum ontology. arXiv:1105.3932 v1 [quant-ph]; http://philsci-archive.pitt.edu/id/eprint/8626. 2011.

[23] Walter Kauzmann. Kinetic Theory of Gases. W. A. Benjamin, Inc., New York, 1966.

[24] Martin Quack. Molecules in motion. Chimia, 55:753-758, 2001.

[25] Charles Hard Townes. The ammonia spectrum and line shapes near 1.25-cm wave-length. Phys. Rev., 70:665-671, 1946.

[26] B. Bleaney and J. H. N. Loubser. The inversion spectra of $\mathrm{NH}_{3}, \mathrm{CH}_{3} \mathrm{Cl}$ and $\mathrm{CH}_{3} \mathrm{Br}$ at high pressures. Proc. Phys. Soc. London A, 63:483-493, 1950.

[27] A. S. Wightman. Superselection rules; old and new. Nuovo Cim. B, 110:751-769, 1995.

[28] G. Birnbaum and A. A. Maryott. Change in the inversion spectrum of $\mathrm{ND}_{3}$ from resonant to nonresonant absorption. Phys. Rev., 92:270-273, 1953.

[29] Giovanni Jona-Lasinio, Carlo Presilla, and Cristina Toninelli. Interaction induced localization in a gas of pyramidal molecules. Phys. Rev. Lett., 88:123001, 2002.

[30] V. Grecchi and A. Sacchetti. Critical conditions for a stable molecular structure. J. Phys. A, 37:35272534, 2004.

[31] Isabel Gonzalo and Pedro Bargueño. Stabilization of chiral molecules by decoherence and environment interactions in the gas phase. Phys. Chem. Chem. Phys., 13:17130-17134, 2011.

[32] A. Ben-Reuven. Transition from resonant to nonresonant line shape in microwave absorption. Phys. Rev. Lett., 14:349-351, 1965.

[33] A. Ben-Reuven. Impact broadening of microwave spectra. Phys. Rev., 145:7-22, 1966.

[34] I. M. Herbauts and D. J. Dunstan. Quantum molecular dynamics study of the pressure dependence of the ammonia inversion transition. Phys. Rev. A, 76:062506, 2007.

[35] Mohammad Bahrami and Angelo Bassi. Tunneling properties of non-planar molecules in a gas medium. Phys. Rev. A, 84:062115, 2011. arXiv:1110.3285v1 [cond-mat.stat-mech].

[36] J. H. Van Vleck and V. F. Weisskopf. On the shape of collision-broadened lines. Rev. Mod. Phys., 17, 227-236, 1945.

[37] Kiruthika Devaraj, Paul G. Steffes, and Bryan M. Karpowicz. Reconciling the centimeter- and millimeter-wavelength ammonia absorption spectra under jovian conditions: Extensive millimeterwavelength measurements and a consistent model. Icarus, 212:224-235, 2011.

[38] Martin Quack. How important is parity violation for molecular and biomolecular chirality? Angew. Chem. Int. Ed. Engl., 41:4618-460, 2002. 